\title{
A Study of Impact of Aptitude in Mathematics as Stream Selection at Higher Secondary Level
}

\author{
PREETAM PYARI, KRITIKA MISHRA AND BINTI DUA
}

Research Scholar, Faculty of Education Dayalbagh Educational Institute (Deemed University), Agra-282005, Uttar Pradesh-INDIA

Email: p.preetam03@gmail.com

Received: August 1, 2016| Revised: August 15, 2016| Accepted: August 26, 2016

Published online: September 05, 2016

The Author(s) 2016. This article is published with open access at www.chitkara.edu.in/publications

\begin{abstract}
Present study explores the aptitude among higher secondary students. It is aimed to find out the effect of stream selection in terms of aptitude. A sample of 100 students has been taken from a government senior secondary school of Agra, Uttar Pradesh-India. Data is collected by administering Differential Aptitude Test (DAT). Result showed that there is found significant effect of aptitude in the stream selection process of the students and for mathematics numerical reasoning, clerical speed and accuracy, mechanical and verbal reasoning constitute the significant role for mathematics as careers option at higher secondary level.
\end{abstract}

Keywords: Aptitude, Mathematics, Higher Secondary Level

\section{INTRODUCTION}

Career is very important aspect of student's life. When student starts choosing stream after $10^{\text {th }}$ class that stream defines their future. Adolescence is the phase of shaping ones personality with high ideals. It is very sensitive age of their lives where they have to take decision for their future. Higher secondary education serves as a foundation for future education and initial direction path for university or higher education. Thus at this stage it is very essential to identify and understand their capabilities to make them aware of that, which options or subjects are suitable for them according to their own efficiency. Higher secondary stage is very crucial stage. At higher secondary stage one has to choose the subjects according to their choices which ultimately influence the future opportunities. Choosing a particular stream right at the beginning has a long lasting impact on student's future. When students start

Issues and Ideas in Education Vol - 4, No - 2 September 2016 pp. 141-149 
Pyari, $P$

Mishra, K

Dua, B

from class $11^{\text {th }}$ they are begins to understand about their interest more clearly and starts journey to specialize in a particular field. This happens only when the subject of interest is the same as the subject for which a student has the required aptitude and personality as well. Therefore, if a student is in the appropriate stream, then it can be used as a building block to start their career and understand the basic concepts associated with it.

Stream selection is very complicated process. So it should be carrier oriented. It is basically includes a process of curious thoughts, mental conflicts, mental pressure and decision making regarding the choice of proper subject selection. The factors which affect the stream selection are: Aptitude, Academic achievement, locus of control, self-assessment of ability, vocational awareness, aptitude, gender and interest in the subject etc. Thus choosing mathematics as a career oriented stream will open the doors of many opportunities in different areas. Mathematics education trains student to make and use measurement, includes the study of computer program, algebra, statistics, geometry, calculus etc.

\section{"A person with an aptitude for mathematics is able to learn mathematical skills with relative ease."}

\section{DEFINITION OF APTITUDE}

Aptitude can be innate, acquired, learned or development component. Aptitude can be defined as the quality that characterized person's way of behavior which serves to indicate how well he/ she can learn to meet \& solve a certain specified kind of problem. Freeman (1971) has defined as "An aptitude is a combination of characteristics indicative of an individual's capacity to acquire (with training) some specific knowledge skill, or a set of organized responses, such as the ability to speak a language, to become a musician, to do a mechanical work". Aptitude plays a major role in child achievement. An individual might have a higher degree of aptitude for type of work and not for certain other. Thus people have wide range of aptitudes. For Example: A student might be good in mathematics but may or may not in literature or music etc. Thus aptitude and achievement are considered as the pillars of stream selection and future career options. Every individual is unique. Everyone differ from another in human activity like leadership, music, art, mechanical, teaching, medical, Engineering and Creativity. These differences can be accessed by different abilities present in the people. There are different types of aptitudes, viz., mechanical, musical, clerical, art, scholastic, law, teaching, medicine, engineering, scientific, numerical, word fluency, special relations aptitudes, etc. Psychologists have 
developed many aptitude tests to measure these aptitudes. General aptitude tests are-Differential Aptitude Test (DAT), General Aptitude Test Battery (GATB), Guilford-Zimmerman Aptitude Survey and the Comprehensive Ability Battery (CAB) developed by Sheridian psychological services of USA.

Differential Aptitude Test (DAT) was used in the present study. It is multifactor test battery of eight aptitude tests. These eight aptitude tests are: (1) Verbal Reasoning (VR), (2) Numerical Reasoning (NA), (3) Language usage-spelling (LU-sp),(4) Language usage-grammar (LU-gr),(5) Clerical speed and accuracy (CSA), (6) Mechanical Reasoning (MR), (7)Abstract Reasoning and(8)Space Reasoning (SR). Each selection consists of twenty questions and ten minute duration for each section was given to the students to complete each section. Differential Aptitude Test (DAT) was combined form of many standardized tools. Aptitudes that are mentioned in it is transformed into Indian circumstances.

Aptitude required for mathematics stream:

\section{Mathematics: Verbal Reasoning + Numerical Reasoning+ Mechanical Reasoning +Abstract Reasoning + Clerical Speed and Accuracy}

\section{RELATED LITERATURE}

The present study is supported with different studies. Tella, A. (2005) studied the impact of motivation on students' Academic Achievement in Mathematics shows that significant difference when extent of motivation was taken as variable of interest on Academic Achievement in Mathematics based on the degree of their motivation [5]. In the other study James et.al (2006) studied that size of effect is influenced by the ability level of the population studied; gains were large for subjects of high ability than they were for subject of low ability [1]. Luz Stenberg et.al (2010) study explored that student's mathematical ability \& attitude played an important role in determining the failure rate in quantitative methods for business at university level [4]. R. A. Olatoye and A. A. Aderogba (2011) indicate that the highest positive significant relationship in between student performance in verbal and numerical ability [2]. Sabahat Usman (2012) in his research study focusing on factor influencing the academic performance of the students and finding reveals that good study environment, regularity in class, Teachers good behavior and proper methodology enhance students achievement[6]. Rajnish Pandey (2012) conducted a research on mathematical aptitude in relation to intelligence and SES findings shows that the mathematical aptitude of the students is significantly related with their intelligence [3].
A Study of Impact
of Aptitude in
Mathematics as Stream Selection at Higher Secondary
Level

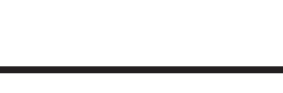


Pyari, $P$

Mishra, K

Dua, B

\subsection{Objective}

To study the effect of aptitude in Mathematics as stream selection at higher secondary level

\subsection{Hypothesis}

There will be no significant effect of aptitude in mathematics stream selection at higher secondary level.

\subsection{Methodology}

Descriptive survey method was used by the researcher in order to consider the research work.

\subsection{Tools}

Differential aptitude test was used by the researcher to measure the aptitude of students towards mathematics as a stream selection at higher secondary level.

\subsection{Sample of the Study}

The sample was 100 students of U.P. board studying in higher secondary level. Random sampling method was used by the researcher. Five schools were randomly selected. From each school 20 students i.e. 10boys and 10 girls were selected who has opted mathematics as their career option.

\subsection{Findings}

First of all mean was calculated from the marks obtained by students in all the sections of the tool including Verbal Reasoning (V.R.), Numerical Reasoning (N.R.), Language Spelling (L.S.), Language Grammar (L. G.), Clerical Speed and Accuracy (C.S.), Mechanical Reasoning (M.R.), Abstract Reasoning (A.R), and Spatial Aptitude (S.A.).The table 1.1 showed the mean and standard deviation values obtained from the scores of students of mathematics stream.

Researcher defines the percentile ranges i.e. $66^{\text {th }}$ percentile and $33^{\text {rd }}$ percentile according to this categorize students in three different categories.

On the basis of above table the percentage of students who lied in high, middle and low levels of different aptitude.

The above table showed the analyzed data representing the percentage value of students of higher secondary level from Mathematics stream out of the strengths of 100 students. The table indicated that the student scores significantly in various sections of the test such as Numerical Reasoning, 
Table 1.1: Mean and S.D. values of Aptitude for Mathematics Streams.

\begin{tabular}{ccccccccc}
\hline Stream & V.R & N.R & L.S & L.G & C.S & M.R & A.R & S.P \\
\hline Mean & 14.82 & 14.22 & 11.82 & 11.02 & 17.5 & 12.3 & 8.7 & 7.75 \\
S.D & 2.5 & 3.61 & 2.9 & 2.07 & 4.58 & 3.05 & 3.8 & 3.7 \\
\hline
\end{tabular}

A Study of Impact of Aptitude in Mathematics as Stream Selection at Higher Secondary Level

Abstract Reasoning, Clerical speed and accuracy, Spatial Aptitude and Mechanical Reasoning. The table showed that the students had abilities in

Table 1.2: Percentile Categorization of Students.

\begin{tabular}{ll}
\hline Percentiles & Categorization of students \\
\hline Above $66^{\text {th }}$ Percentile & Percentage of students having high level of aptitude \\
Between $66-33^{\text {rd }}$ percentile & Percentage of students having medium level of aptitude \\
Below $33^{\text {rd }}$ Percentiles & Percentage of students having low level of aptitude \\
\hline
\end{tabular}

allied aptitudes like Numerical Reasoning and clerical speed and accuracy, abstract Reasoning and Spatial Aptitude. The results as presented in the table

Table 1.3: Percentage of High, Middle and Low level of students of Mathematics Stream in different Aptitude.

\begin{tabular}{llll}
\hline \multirow{2}{*}{ Aptitudes } & \multicolumn{2}{l}{ Percentage of Students } \\
\cline { 2 - 4 } & High & Middle & Low \\
\hline Verbal Reasoning & $45 \%$ & $43 \%$ & $12 \%$ \\
Numerical Reasoning & $65 \%$ & $27 \%$ & $8 \%$ \\
Language -Spelling & $43 \%$ & $38 \%$ & $19 \%$ \\
Language-Grammar & $39 \%$ & $53.5 \%$ & $7.5 \%$ \\
Clerical Speed and Accuracy & $72.5 \%$ & $15 \%$ & $12.5 \%$ \\
Mechanical Reasoning & $65 \%$ & $23 \%$ & $12 \%$ \\
Abstract Reasoning & $50 \%$ & $27 \%$ & $23 \%$ \\
Spatial Aptitude & $49 \%$ & $26 \%$ & $25 \%$ \\
\hline
\end{tabular}


Pyari, $\mathrm{P}$ Mishra, K Dua, B depicted that the students had the aptitudes suitable for this stream. Still there is scope of improvement in the section of verbal Reasoning. Hence they would be able to gain positively in the stream they opted for their future career.

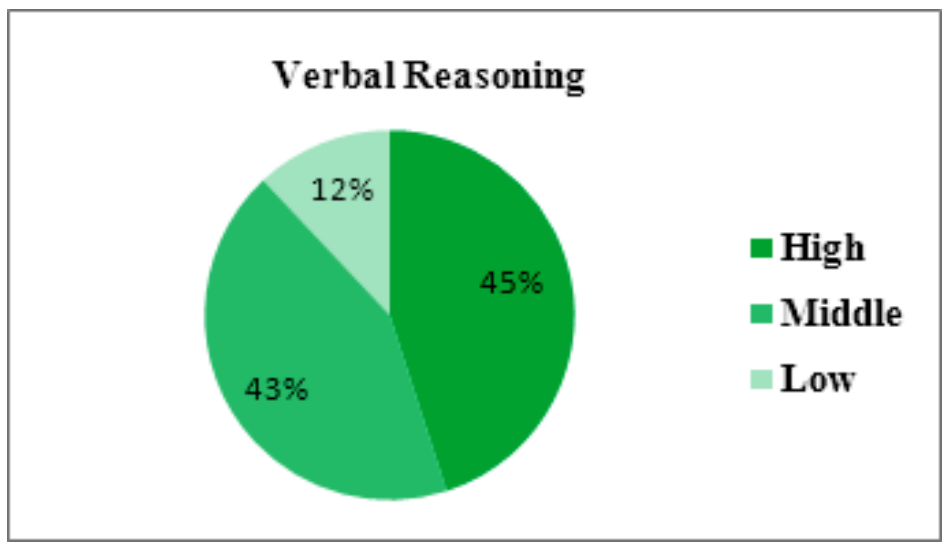

Figure (a)

The Figure (a) of Verbal Reasoning showed that out of 100 students of mathematics stream $45 \%$ Students had high level of verbal reasoning and $12 \%$ students had low level of verbal reasoning.

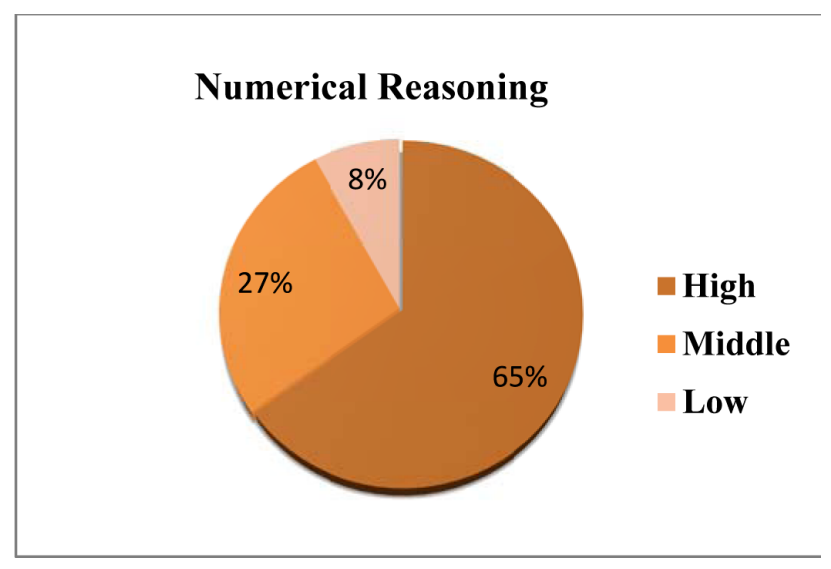

Figure (b)

The Figure (b) of Numerical Reasoning showed that out of 100 students of mathematics stream $65 \%$ Students had high level of numerical reasoning and $8 \%$ students had low level of numerical reasoning. 


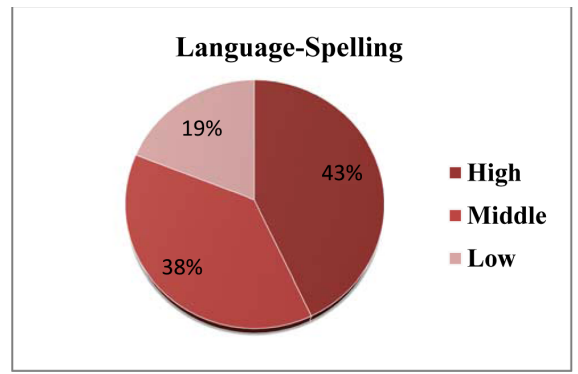

A Study of Impact of Aptitude in

Mathematics as

Stream Selection at

Higher Secondary

Level

Figure (c)

The Figure (c) of Language Spelling showed that out of 100 students of mathematics stream $43 \%$ Students had high level of Language Spelling and $19 \%$ students had low level of Language Spelling which doesn't constitute an important function of mathematics stream selection.

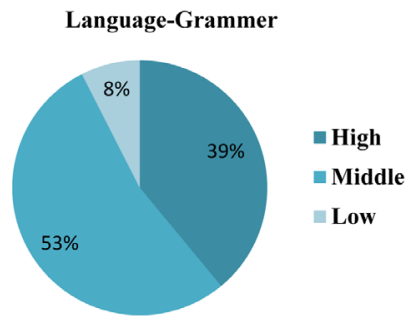

Figure (d)

The Figure (d) of Language Grammar showed that out of 100 students of mathematics stream 54\% Students had high level of Language Grammar and 9 \%students had low level of Language Grammar which also doesn't constitute an important function of mathematics stream selection.

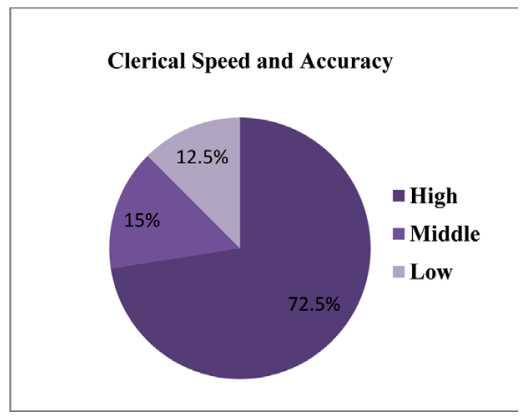

Figure (e)

The Figure (e) of Clerical speed and accuracy showed that out of 100 students of mathematics stream 72.5\% Students had high level and $12.5 \%$ students had low level of Clerical speed and accuracy which constitute an important function of mathematics stream selection. 
Pyari, $\mathrm{P}$

Mishra, K

Dua, B

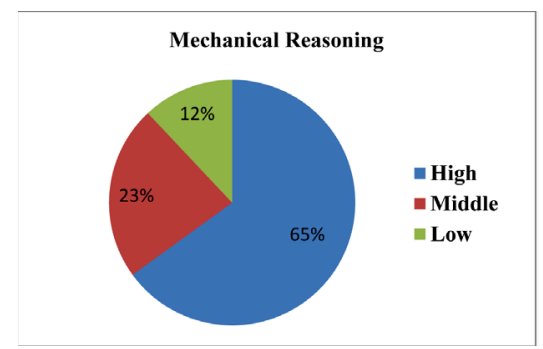

Figure (f)

The Figure (f) of Mechanical Reasoning showed that out of 100 students of mathematics stream 65\% Students had high level of Mechanical Reasoning and $12 \%$ students had low level of Mechanical Reasoning which plays a significant role in mathematics stream selection.

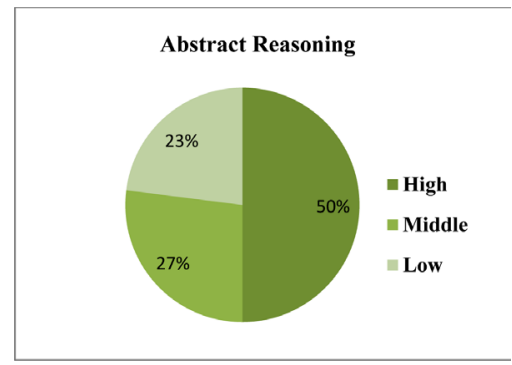

Figure (g)

The Figure (g) of Abstract Reasoning showed that out of 100 students of mathematics stream 50\% Students had high level of Abstract Reasoning and $23 \%$ students had low level of Abstract Reasoning which constitute an important function of mathematics stream selection.

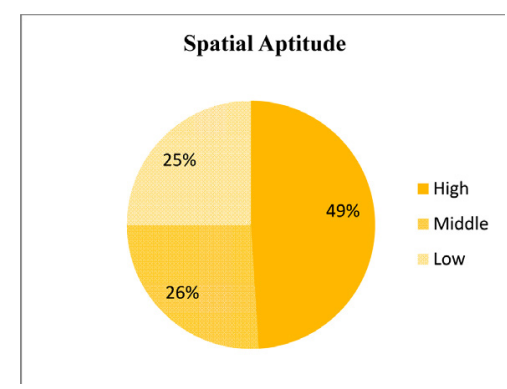

Figure (h)

The Figure (h) of Spatial Aptitude showed that out of 100 students of mathematics stream 49\% Students had high level of spatial reasoning and $25 \%$ students had low level of Abstract Reasoning which constitute an important function of mathematics stream selection. 


\section{CONCLUSION}

It concludes that Mathematics stream includes high level of aptitudes like numerical reasoning, mechanical reasoning and abstract reasoning. It had medium level of aptitude like language-grammar, clerical speed and accuracy and spatial aptitude. It also achieved low level in the aptitudes like verbal reasoning and language-spelling. Thus for mathematics as a stream selection numerical reasoning, mechanical reasoning, clerical speed and accuracy, spatial ability, verbal reasoning and abstract reasoning plays a significant role. Today's students are much more aware about their abilities, interest and aptitudes. But still there is a need to effective efforts by teachers and society to aware the students to choose appropriate stream at higher secondary level.

\section{REFERENCES}

[1] Kulik, J.A., Kulik, C.C.\& Bangert, R.L., (2006). Effect of practice on aptitude and Achievement test scores. American Educational Research Journal, 21(2), 435-447. Retrieved from http://www.jstor.org/stable/1162453 https://doi.org/10.3102/00028312021002435

[2] Olatoye, R.A. \& Aderogba, A.A., (2011). Performance of Senior Secondary school science students in aptitude test: the role of student's verbal and numerical abilities. Journal of Emerging Trends in Educational Research and Policy Studies, 2(6), 431-435. Retrieved from jeteraps.scholarlinkresearch.org

[3] Pandey, R., (2012). A study of mathematical aptitude in relation to intelligence and SES of class VI students. Indian Journal of Education Research experimentation and innovation, 2(2)

[4] Stenberg, L. C., Varua, M. E., \& Yong, J. (2010). Mathematics Aptitude, Attitude, Secondary Schools and Student Success in Quantitative Methods for Business Subject in an Australian Catholic University Experience.39th Australian Conference of Economists. Retrieved from http://researchonline.nd.edu.au/bus_conference/26

[5] Tella, A. (2007). The impact of motivation on students' Academic Achievement and learning outcomes in mathematics among secondary school students in Nigeria. Eurasia Journal of Mathematics, Science \& Technology Education, 3(2), 149-156.

[6] Usman, S., (2012). Factor influencing students' Academic performance at Higher Secondary Level. Retrieved from www.languageinindia.com
A Study of Impact of Aptitude in

Mathematics as

Stream Selection at

Higher Secondary

Level

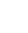

